Michel Pereira Santos ${ }^{1}$

Viviane Cristina Sebben ${ }^{2}$

Paulo Roberto Farenzena ${ }^{3}$

Celso Felipe Dexheimer ${ }^{4}$

Cláudia Pereira Santos ${ }^{5}$

Vera Maria Steffen ${ }^{6}$

\title{
Exposição a agentes químicos e ruído em indústria de couro
}

\author{
Exposure to chemical agents and noise in \\ tanneries
}

${ }^{1}$ Farmacêutico Discente do Curso de Análises Clínicas da Faculdade de Farmácia da Universidade Federal do Rio Grande do Sul (UFRGS).

${ }^{2}$ Farmacêutica-Bioquímica do Centro de Informação Toxicológica(CIT/RS)/FEPPS.

${ }^{3}$ Médico Otorrinolaringologista do Centro de Diagnósticos do Hospital Divina Providência.

${ }^{4}$ Farmacêutico-Bioquímico do Laboratório Pró-Ambiente-Análises Químicas e Toxicológicas.

${ }^{5}$ Fonoaudióloga da Clínica Audiomed Audiologia e Medicina do Trabalho.

${ }^{6}$ Doutora do Departamento de Análises da Faculdade de Farmácia da

Universidade Federal do Rio Grande do Sul (UFRGS).

\begin{abstract}
Resumo
Este estudo tem como objetivo investigar a relação entre a perda auditiva e a exposição ocupacional ao ruído e ao tolueno. A população em estudo foi composta por 73 trabalhadores de curtume. Para conhecer a história clínica e ocupacional dos trabalhadores, aplicou-se um questionário. Para avaliação da exposição ocupacional, realizaram-se avaliações ambiental e biológica do tolueno e avaliações audiológica e dos níveis de ruído. Os valores obtidos na avaliação ambiental e biológica estavam abaixo dos limites estabelecidos pelas NR 7 e 15. Os níveis de ruído em diversos setores da indústria foram superiores ao máximo permitido pela NR 15, chegando a $97.8 \mathrm{~dB}(\mathrm{~A})$. As perdas auditivas encontradas no grupo ruído (GR) e no grupo ruído e agente químico (GRAQ) foram significativas quando comparadas ao grupo controle através da análise estatística SPSS ${ }^{\circledR}$, T-Test $p<0,01$. Este estudo demonstrou fatores de impacto na saúde e na qualidade de vida dos trabalhadores. Portanto, fazem-se necessárias a revisão dos programas e a implementação de medidas que reduzam os riscos de forma a prevenir e evitar danos à saúde do trabalhador.
\end{abstract}

Palavras-chave: tolueno, ruído, avaliação ambiental, audiometria, avaliação biológica.

\begin{abstract}
This study aims at investigating the relationship between the hearing loss and the occupational exposition to noise and toluene. Seventy-three tannery workers were the subject of this study. A questionnaire was used to obtain information on the workers' clinical and occupational history. To evaluate the occupational exposition, an environmental and biological survey on toluene was held, together with noise and audiologycal evaluations. The results of the environmental and biological evaluations were below the levels established by NR 7 and 15. The noise levels in several sections of the industry were above the maximum allowed by NR 15, reaching $97.8 d B(A)$. The hearing losses found in the noise group (GR) and noise and chemical agents (GRAQ) groups were significant when compared, through the statistic analysis SPSS ${ }^{\circledast}, T$-Test $p<0,01$, to the control group. This study demonstrated impact factors in workers' health and life quality. Due to this, the programs must be revised and measures to reduce the risks must be implemented in order to prevent hazards to workers' health.
\end{abstract}

Keywords: toluene, noise, environmental evaluation, audiometry, biological evaluation. 


\section{Introdução}

Maior precisão, sensibilidade e alcance que qualquer outro órgão do sentido - essas são as qualidades que se pode atribuir à orelha (Santos et al., 1996). A audição é um sentido importante do qual nenhum ser humano deveria ser privado. Atualmente, a intensa produção de ruído, proveniente principalmente das indústrias, tem contribuído em muitos casos de alterações auditivas. Cresce, assim, a cada dia, o número de perdas auditivas induzidas pelo ruído industrial, hipoacusias que poderiam ser reduzidas através da prevenção e do esclarecimento sobre os efeitos do ruído.

Diversos estudos, principalmente no campo da toxicologia ocupacional, vêm colocando em evidência que os danos auditivos não se limitam apenas à ação do ruído. Recentes descobertas sugerem o sinergismo entre o ruído e diversos agentes químicos, como tolueno, metais e outros produtos encontrados em muitas situações nos ambientes de trabalho. (Barregard e Axelsson, 1984; Fiorini et al., 1998; Jacobsen et al., 1993; Morata et al., 1993, 1994, 1997). Por esse motivo, a expressão Perda Auditiva Ocupacional não deveria restringir-se a um sinônimo da expressão Perda Auditiva Induzida por Ruído (PAIRO), uma

\section{Materiais e Métodos}

\section{População}

Este estudo foi aprovado pelo Comitê de Ética da Fundação Estadual de Produção e Pesquisa em Saúde do Estado do Rio Grande do Sul e realizado em uma indústria de curtimento de couro, localizada na região metropolitana de Porto Alegre, Rio Grande do Sul, Brasil. Participaram voluntariamente do estudo 73 trabalhadores que cumprem uma jornada de trabalho diária de 8 horas em período diurno $(n=64)$ ou noturno $(n=9)$ e possuem tempo de serviço no setor de couros superior a um ano, sendo que os mesmos permanecem em suas determinadas funções e setores sem haver alteração em suas dinâmicas de trabalho (salvo as ocorrências vez que podem ocorrer casos de perdas auditivas ocupacionais independentes da exposição ao ruído (Morata e Dunn, 1995; Morata et al., 1995).

Os trabalhadores de indústria de curtimento de couro (curtume) estão freqüentemente expostos a produtos que contêm cromo, anilinas e tolueno. Este último é usado principalmente como solvente para colas, óleos, resinas e diluente para tintas e vernizes. Esse hidrocarboneto aromático pode provocar diversas ações tóxicas no organismo, como a perda auditiva neurossensorial. Embora o mecanismo das lesões danosas à audição não tenha sido completamente entendido, aceitase que o tolueno altera a função auditiva dos humanos (Morata et al., 1997).

A ação tóxica das substâncias presentes no ambiente industrial, a presença de elevados níveis de ruído aliados à necessidade de se evitar que ocorram perdas auditivas nos trabalhadores expostos motivaram o presente estudo. Este trabalho teve como objetivo investigar a relação entre a perda auditiva e a exposição ocupacional ao ruído e ao tolueno presentes no ambiente laboral da indústria de curtimento de couro. de situações emergenciais por determinados fatores como, por exemplo, agravamentos de perdas auditivas, em que ocorrem mudanças de funções determinadas pelo médico do trabalho coordenador do programa de controle médico de saúde ocupacional (PCMSO)). Os trabalhadores, após a apresentação e os esclarecimentos sobre o estudo, assinaram o termo de consentimento informado. Aplicouse um questionário composto por 39 questões referentes à história clínica e ocupacional, ao estilo de vida e à descrição da função executada.

Após a avaliação ambiental e análise dos níveis de ruído, os trabalhadores foram divididos em grupos (vide Tabela 1).

Tabela 1 Definição dos grupos

\begin{tabular}{cccc}
\hline $\boldsymbol{G C}$ & $\boldsymbol{G R}$ & $\boldsymbol{G R A Q}$ & $\boldsymbol{G A Q}$ \\
\hline Grupo controle & Grupo ruído & Grupo ruído agente químico & Grupo agente químico \\
$\begin{array}{c}\text { Trabalhadores não } \\
\text { expostos a ruído } \\
\text { e agente químico }\end{array}$ & $\begin{array}{c}\text { Trabalhadores expostos } \\
\text { somente ao ruído }\end{array}$ & $\begin{array}{c}\text { Trabalhadores expostos } \\
\text { a ruído (valores acima } \\
\text { de } 85 \mathrm{~dB}(\mathrm{~A})) \text { e à presença } \\
\text { de tolueno no ambiente }\end{array}$ & $\begin{array}{c}\text { Trabalhadores expostos } \\
\text { a níveis de ruído abaixo } \\
\text { de 85 dB(A) à presença de } \\
\text { tolueno no ambiente }\end{array}$ \\
\hline
\end{tabular}




\section{Avaliação ambiental}

Os agentes físicos e químicos objeto deste estudo foram definidos através do fluxograma de produção da indústria.

A avaliação dos níveis de ruído no ambiente laboral foi realizada seguindo os procedimentos indicados na norma brasileira NBR-10151 (Associação Brasileira de Normas Técnicas, 2000). O equipamento utilizado foi o medidor de nível de pressão sonora modelo $\mathrm{n}^{\circ}$ 886-2 (Simpson/EUA) de acordo com os padrões da ANSI S1.4-1983.

A avaliação ambiental do tolueno foi realizada por Cromatografia a Gás com Detector de Massas, segundo NIOSH 1501 (National Institute for Occupational Safety and Health, 2003a). A estratégia de amostragem foi dois trabalhadores para cada setor da empresa. O tipo de coletor utilizado foi o Tubo de Carvão de Casca de Coco de 50/ $100 \mathrm{mg}$, com vazão da bomba / volume de ar coletado: 0,0438 L/min / 3,066 L.

\section{Avaliação biológica}

Foi realizada através da análise do ácido hipúrico, indicador biológico de exposição (IBE) ao tolueno. As amostras de material biológico foram coletadas conforme previsto pela NR 7 (Associação Brasileira para Prevenção de Acidentes, 1998a). As amostras de urina foram coletadas em recipientes de PVC, no início da jornada de trabalho (entre $6 \mathrm{~h}$ e $7 \mathrm{~h}$ no período diurno e entre $18 \mathrm{~h}$ e $19 \mathrm{~h}$ no período noturno) e imediatamente após seu final (entre 17h20min e 18h20min e entre $5 \mathrm{~h} 20 \mathrm{~min}$ e $6 \mathrm{~h} 40 \mathrm{~min}$, respectivamente), evitando-se a primeira jornada da semana. As amostras foram mantidas sob refrigeração entre $2^{\circ}$ e $8^{\circ} \mathrm{C}$ para preservação durante o intervalo de tempo entre a coleta e a análise (inferior às 24h).

O método de análise do ácido hipúrico consistiu em preparar as amostras conforme

\section{Resultados}

\section{Avaliação do questionário}

Foram relatadas várias enfermidades como problemas respiratórios, hepáticos, neurológicos, circulatórios, renais, gastrintestinais, anemia, alteração no olfato e sangramento nasal, ulcerações e perfurações de septo nasal, feridas nas mãos e dedos e hipertensão.

O uso diário de medicamentos foi referido por $9,6 \%$ dos trabalhadores. Segundo a sugerido por Roosmalen e Drummond (1978). Utilizou-se o cromatógrafo a gás modelo 3800 (VARIAN/USA) e a coluna CPSIL 8CB. As condições cromatográficas utilizadas foram: fluxo do gás de arraste (He) $1,0 \mathrm{~mL} / \mathrm{min}$.; temperatura do injetor e do detector $230^{\circ} \mathrm{C}$; programação de temperatura da coluna (DB5 30mx0,25 mmx0,25 mm) inicial de $150^{\circ} \mathrm{C}$ por $3 \mathrm{~min}$., rampa de $50^{\circ} \mathrm{C} /$ min. até $190^{\circ} \mathrm{C}$, manutenção por $1,0 \mathrm{~min}$., rampa de $50^{\circ} \mathrm{C} / \mathrm{min}$. até $200^{\circ} \mathrm{C}$, manutenção por $4,0 \mathrm{~min}$.; rampa de $50^{\circ} \mathrm{C} / \mathrm{min}$. até $250^{\circ} \mathrm{C}$, manutenção por 2,5min.; injetor capilar com razão de divisão de 1:50.

\section{Avaliação audiológica}

A avaliação audiológica ocupacional dos trabalhadores foi realizada por via aérea e óssea, após repouso auditivo de 14 horas. Foi utilizada Cabine Audiométrica modelo VSA-40 (Vibrasom/BRA), audiômetro AD 229 (Interacoustics/DIN) devidamente calibrado. O audiograma utilizado e todos os parâmetros de avaliação e calibração seguiram os critérios especificados pela NR 7. As freqüências utilizadas para avaliação audiológica por via aérea foram $500,1 \mathrm{k}, 2 \mathrm{k}, 3 \mathrm{k}, 4 \mathrm{k}, 6 \mathrm{k}$, e $8 \mathrm{k}$ $\mathrm{Hz}$ e para via óssea $500,1 \mathrm{k}, 2 \mathrm{k}, 3 \mathrm{k}, 4 \mathrm{k} \mathrm{Hz}$. Foram realizadas meatoscopias prévias em todos os trabalhadores a fim de descartar eventuais obstruções de canal auditivo externo que poderiam alterar o resultado das audiometrias.

\section{Análise estatística}

Os dados do presente trabalho foram analisados estatisticamente através do programa SPSSÒ, versão 11.5, utilizando a Estatística Descritiva (média, desvio padrão, freqüências, percentuais, máximos e mínimos), Intervalo de Confiança, Análise de Variância (ANOVA - Oneway) e teste-t (de Student). Nas conclusões, utilizou-se o nível de significância de $5 \%$.

literatura, nenhum dos citados influencia nas análises realizadas.

A empresa disponibiliza os EPIs adequados a cada tipo de função e fornece orientação quanto à importância e à forma de utilização dos mesmos. Os trabalhadores expostos ao ruído utilizam protetor auricular do tipo plug há pelo menos um ano; alguns, em determinadas situações, utilizavam-no em uma única orelha. A maioria dos trabalhadores utiliza calçados e botas apropriados, 
luvas, máscaras e óculos, porém nem sempre são utilizados durante todo o expediente. Freqüentemente a utilização ocorre quando os trabalhadores julgam necessário.

\section{Avaliação do fluxograma de produção}

A partir da avaliação do fluxograma de produção da indústria, verificou-se a presença de tolueno como componente de colas, vernizes e tintas; anilinas utilizadas como corantes; cromo como constituinte da marca comercial Tanicor PCS $®[\mathrm{Cr}(\mathrm{OH})(\mathrm{SO})]$ e ruído em pontos específicos da linha de produção, direcionando o estudo para a avaliação dos níveis de ruído e ambiental do tolueno.

\section{Avaliação dos níveis de ruído}

Os níveis de ruído encontrados estão apresentados na Tabela 2. A NR 15 (Associação Brasileira para Prevenção de Acidentes, 1998b) limita em $85 \mathrm{~dB}(\mathrm{~A})$ os níveis de ruído para uma jornada de trabalho de $8 \mathrm{~h} /$ dia $48 \mathrm{~h} /$ semana.

\section{Avaliações ambiental e biológica}

Os resultados obtidos nas avaliações ambiental e biológica estão apresentados na Tabela 2. Os limites de tolerância (LT) estabelecidos pela NR 15 para uma jornada de trabalho semanal de $48 \mathrm{~h}$ para tolueno são 78ppm. O IBMP (índice biológico máximo permitido) descrito na NR 7 para o IBE ácido hipúrico é $2,5 \mathrm{~g} / \mathrm{g}$ creatinina.

Tabela 2 Resultados das avaliações ambiental, biológica, audiológica e do ruído

\begin{tabular}{|c|c|c|c|c|c|c|c|c|c|}
\hline \multirow{2}{*}{ Grupos } & \multicolumn{2}{|c|}{$\begin{array}{l}\text { Tolueno (ppm) } \\
\text { (g/gcreatinina) }\end{array}$} & \multicolumn{2}{|c|}{ Ácido Hipúrico } & \multicolumn{3}{|c|}{ Ruído dB(A) } & \multicolumn{2}{|c|}{ Avaliação Audiológica } \\
\hline & Média \pm SD & LT/NR 15 & Média \pm SD & IBMP/NR 7 & Faixa dos níveis & Média & VR/NR 15 & $n=64$ & PAN \\
\hline GC & ND & & $0,31 \pm 0,05$ & & $55,0-65,0$ & 57,5 & & 8 & 0 \\
\hline \multirow[t]{2}{*}{ GR } & ND & & $0,48 \pm 0,67$ & & $87,4-97,8$ & 92,6 & & 17 & 10 \\
\hline & & 78 & & 22,5 & & & 85 & & \\
\hline GRAQ & $3,30 \pm 0,24$ & & $0,66 \pm 0,69$ & & $86,0-87,8$ & 86,2 & & 17 & 15 \\
\hline GAQ & $2,62 \pm 1,38$ & & $0,66 \pm 0,59$ & & $75,1-84,6$ & 80,1 & & 22 & 0 \\
\hline
\end{tabular}

SD = Desvio Padrão; ND = Não Detectado; LT = Limite Tolerância; IBMP = Índice Biológico Máximo Permitido; VR = Valor de Referência; PAN = Perda Auditiva Neurossensorial; GC = Grupo Controle; GR = Grupo Ruído; GRAQ = Grupo Ruído Agente Químico; GAQ = Grupo Agente Químico

\section{Avaliação audiológica}

Alterações audiológicas foram percebidas em 34 dos 73 trabalhadores. A análise dos dados obtidos através do questionário aliada aos resultados da avaliação audiológica permitiu identificar quais trabalhadores possuiam histórico clínico que pudesse interferir na audição.

Para a análise estatística envolvendo perda auditiva sugestiva de PAIRO, foram excluídos 9 trabalhadores que apresentaram perdas auditivas que não se enquadram nos traçados audiológicos da PAIRO (ex.: perda condutiva, mista) e fatores de exclusão, como diabetes, hipertensão e surdez familiar, resultando em $\mathrm{n}=64$. O número de trabalhadores avaliados em cada grupo e a quantidade de perdas auditivas sugestivas de PAIRO observadas estão apresentados na Tabela 2.

\section{Características da população}

As características da população estudada estão apresentadas na Tabela 3.

Tabela 3 Características da população

\begin{tabular}{cccccccccc}
\hline & & \multicolumn{3}{c}{ Idade (anos) } & \multicolumn{2}{c}{ Sexo } & \multicolumn{2}{c}{ Tempo de Exposição(anos) } \\
\cline { 3 - 9 } Grupos & $\mathbf{n}$ & Média + SD & Min-Max & I.C.* 95\% & M & F & Média + SD & Min-Max & I.C.* 95\% \\
\hline GC & 8 & $36,8 \pm 8,0$ & $23-44$ & $30,1-43,4$ & 3 & 5 & - & - \\
GR & 17 & $35,7 \pm 9,7$ & $21-53$ & $30,7-40,7$ & 16 & 1 & $18,8 \pm 9,6$ & $6,0-36,0$ & $13,9-23,8$ \\
GRAQ & 17 & $39,0 \pm 9,9$ & $23-56$ & $33,9-44,1$ & 17 & 0 & $20,6 \pm 10,2$ & $5,0-38,0$ & $15,4-25,9$ \\
GAQ & 22 & $25,7 \pm 5,4$ & $19-37$ & $23,3-28,1$ & 20 & 2 & $6,3+4.3$ & $1,0-16,0$ & $4,4-8,2$ \\
\hline
\end{tabular}

*Intervalo de confiança 95\%; SD = Desvio Padrão; GC = Grupo Controle; GR = Grupo Ruído; GRAQ = Grupo Ruído Agente Químico; GAQ = Grupo Agente Químico 


\section{Discussão e Conclusões}

Os níveis ambientais do tolueno foram menores que seu respectivo limite de tolerância (LT). Os resultados obtidos na avaliação biológica estão de acordo com os dados da avaliação ambiental, visto que os valores estão abaixo do IBMP.

Os níveis de ruído ao qual os trabalhadores do GR e do GRAQ estão expostos encontramse acima do limite estabelecido pela NR 15 para uma jornada de $8 \mathrm{~h} /$ dia (48h/semana). O GR e o GRAQ, quando comparados ao GC em relação à perda auditiva, apresentaram diferença estatisticamente significativa $(\mathrm{p}<0,01)$. Entre o GR e o GRAQ não houve diferença estatisticamente significativa. Isso pode estar relacionado ao fato dos níveis de ruído entre estes grupos diferir em média 6,4 $\mathrm{dB}(\mathrm{A})$ e também devido aos baixos níveis de tolueno observados. No GR, os níveis de ruído em alguns setores chegaram a 97,8 dB(A). Segundo a NR 15, a exposição diária máxima permissível aos trabalhadores expostos a este nível de ruído é 1h15min por dia. Entretanto, no GRAQ, os níveis de ruído atingiram 87,8 $\mathrm{dB}(\mathrm{A})$, sendo que a exposição máxima a este nível de ruído é $5 \mathrm{~h} /$ dia. Desta forma, os indivíduos do GR sofreram uma perturbação sonora significativamente maior do que os do GRAQ. No GR, observou-se que os indivíduos que ainda não apresentam perda sugestiva de PAIRO são pessoas jovens (20-30 anos) e com pouco tempo de exposição ao ruído. No presente estudo constatou-se que a exposição excessiva ao ruído é a principal causa de perda auditiva na população.

Consideramos importante a realização de exames complementares (glicemia, triglicerídeos, colesterol, uréia, creatinina, T3, T4, TSH, sorologia para Lues) e avaliação da pressão arterial nos trabalhadores com perda auditiva para verificar fatores de agravamento e a possibilidade da mesma estar relacionada a alguma patologia.

O ruído industrial provoca danos não só auditivos, mas tabém afeta vários órgãos e sistemas do organismo. Estes efeitos vão desde alterações passageiras até graves danos irreversíveis. Considerando a exposição ao ruído, a Organização Mundial da Saúde (OMS) estabelece que o estresse auditivo ocorre a partir de 55 dBA de exposição (WHO, 1980). Casos de estresse auditivo crônico podem desencadear distúrbios orgânicos e psicológicos, como náuseas, cefaléia, instabilidade emocional, redução da libido, perda ou aumento de apetite, sonolência, insônia, aumento da prevalência de úlcera, hipertensão arterial, tontura, vertigem e desatenção (Andrade et al., 1998).

$\mathrm{Na}$ Alemanha constatou-se maior incidência de hipertensão e distúrbios circulatórios em trabalhadores de fábricas barulhentas. As populações submetidas a níveis entre 65 e 70 dBA tiveram de $10 \%$ a $20 \%$ mais infarto e, entre 70 e $80 \mathrm{dBA}, 20 \%$ (GERGES, 1993). Assim, o ruído pode ter contribuído para o aparecimento e/ou agravamento de algumas das manifestações patológicas apresentadas pelos trabalhadores.

Além das avaliações ambiental, biológica, audiológica e dos níveis de ruído, é de extrema importância a conscientização dos trabalhadores com relação à utilização dos EPIs e a implementação de equipamentos de proteção coletiva (EPCs). O trabalhador influenciado por fatores ambientais inadequados reduz sua produtividade e sofre maior número de acidentes de trabalho (Santos et al., 1996). A inadequação do ambiente ocupacional coloca em risco a segurança e o bem-estar dos trabalhadores, portanto, fazem-se necessárias a revisão de programas e a implementação de medidas que reduzam os riscos de forma a prevenir e evitar danos à saúde do trabalhador. Uma fiscalização eficaz pode impedir que as não conformidades possam motivar a ocorrência de acidentes de trabalho e doenças ocupacionais.

\section{Agradecimentos}

Os autores agradecem à Sra. Jurema Enzweiller, aos funcionários do Núcleo de Análise Laboratorial do Centro de Informação Toxicológica (CIT/RS), ao laboratório Pró-Ambiente e à empresa por permitir a realização do estudo.

Apoio: $\mathbf{C N P q}$ 


\section{Referências Bibliográficas}

ANDRADE, D. R. de. et al. Efeitos do ruído industrial no organismo. Pró-Fono, v.10, n.1, p.17-20, 1998.

ASSOCIAÇÃO BRASILEIRA DE NORMAS TÉCNICAS. NBR10151: Acústica - Avaliação do Ruído em Áreas Habitadas, Visando o Conforto da Comunidade - Procedimento. Rio de Janeiro: ABNT, 2000.

ASSOCIAÇÃO BRASILEIRA PARA PREVENÇÃO DE ACIDENTES. NR-7 Programa de Controle Médico de Saúde Ocupacional. In: Legislação de Segurança e Medicina do Trabalho. Rio de Janeiro: ABPA, 1998a.

NR-15 - Atividades e Operações insalubres. In: Legislação de Segurança e Medicina do Trabalho. Rio de Janeiro: ABPA, 1998b.

BARREGARD, L.; AXELSSON, A. Is there an ototraumatic interaction between noise and solvents? Scand. Audiol. v. 13, p.151-155, 1984.

FIORINI, A. C. et al. Efeitos da interação ruído e solventes na audição de trabalhadores gráficos. In: I CONGRESSO IBEROAMERICANO DE ACÚSTICA, Florianópolis. Anais, 1998. p. 222-230.

GERGES, S. N. Y. Inimigo Invisível. Revista Proteção. v. 5, p. 74-77, 1993.

JACOBSEN, P. et al. Mixed solvent exposure and hearing impairment: an epidemiological study of 3284 men. The Copenhagen male study. Occupational Medicine. v.43, n.4, p.180-184, 1993.

MORATA, T. C.; DUNN, D. E. Occupational hearing loss. Ocupational Medicine: State of the Art Reviews, v.10, n.3, 1995.
MORATA T. C. et al. Effects of occupational exposure to organic solvents and noise on hearing. Scand. J. Work Environ. Health. v.19, n.4, p. 245-254, 1993.

MORATA, T. C.; DUNN D. E.; SIEBER, W. K. Occupational exposure to noise and ototóxic organic solvents. Arch Environ Health, v.49, n.5, p. 359-365, 1994.

- Perda auditiva e a exposição ocupacional a agentes ototóxicos. In: NUDELMANN, A. A.; COSTA, E. A.; SELIGMAN, J.; IBANEEZ, R. N. PAIR - Perda Auditiva Induzida pelo Ruído. Porto Alegre: Bagaggem Comunicação, 1997.

MORATA, T. C.; NYLÉN, P. R.; JOHNSON, A. C.; DUNN, D. E. Auditory and vestibular functions after single or combined exposure to toluene: a review. Archives of Toxicology. v. 69, p. 413-443, 1995.

NATIONAL INSTITUTE FOR OCCUPATIONAL SAFETY AND HEALTH. Manual of Analytical Methods (NMAM). $4 \mathrm{ed}$. Cincinnati: U.S. Department of Health and Human Services, 2003a. Method 1501. Disponível em <http://www.cdc.gov/niosh/ nmam/pdfs/1501.pdf $>$ Acesso em 15 maio 2004.

ROOSMALEN, P. Van; DRUMMOND, I. Simultaneous determination by gas chromatography of the major metabolites in urine of toluene, xilene and styrene. British Journal of Industrial Medicine. v. 35, p.5660, 1978.

SANTOS, U. P. (org.) Ruído, riscos e prevenção. 2 ed. São Paulo: Hucitec, 1996.

World Health Organization (1980): Noise. Geneve: WHO. 103 p. 